\title{
CARACTERIZACIÓN QUÍMICA Y CLASIFICACIÓN TAXONÓMICA DE ALGUNOS SUELOS CULTIVADOS CON BANANO EN LAS LLANURAS ALUVIALES DEL CARIBE DE COSTA RICA
}

\author{
Fulvio Arias $^{1 / *}$, Rafael Mata**, Alfredo Alvarado ${ }^{* *}$, Edgardo Serrano $^{* * *}$, Jorge Laguna $^{* * * * *}$ \\ Palabras clave: Clasificación suelos, Inceptisoles, Andisoles, química suelos, fertilidad suelos, banano, Costa Rica. \\ Keywords: Soil taxonomy, Inceptisols, Andisols, soil chemistry, soil fertility, banana, Costa Rica.
}

Recibido: 17/11/09

Aceptado: $15 / 04 / 10$

\section{RESUMEN}

Se realizó la caracterización morfológica, química y la clasificación taxonómica de algunos suelos cultivados con banano en las 11 cuencas hidrográficas que confluyen en las llanuras aluviales de la Vertiente Caribe de Costa Rica. Se identificaron 2 sectores con suelos que tienen propiedades morfológicas, químicas, y taxonómicas diferentes. En una zona, se encontraron principalmente Inceptisoles formados a partir de materiales fluvio-volcánicos con propiedades ándicas (Andic Eutrudepts, Aquandic Endoaquepts), Inceptisoles dístricos (Fluventic Dystrudepts) y Andisoles (Typic Hapludands); caracterizados por presentar la mayoría buen drenaje, texturas medias (franco arenosa, franca, franca limosa), una suma de bases entre 10 y 19 cmol. $\mathrm{l}^{-1}$, una CIC entre 13 y $28 \mathrm{cmol}^{-1} \mathrm{l}^{-1}$ y carga permanente en la arcillas. En la otra zona, se encontraron principalmente Inceptisoles formados a partir de aluviones de origen sedimentario depositados por inundaciones frecuentes (Fluventic Endoaquepts, Dystric Fluventic Eutrudepts, Fluvaquentic Eutrudepts, Fluvaquentic Endoaquepts), con una suma de bases entre $18 \mathrm{y}$ $46 \mathrm{cmol}^{-1}$, una CIC entre 19 y $46 \mathrm{cmol}^{-1} \mathrm{C}^{-1}$, sin propiedades ándicas, texturas finas y moderadamente finas (franco arcillosa, franco arcillosa

1 Autor para correspondencia. Correo electrónico: farias@delorocr.com

* Grupo del ORO. La Cruz, Guanacaste, Costa Rica.

** Centro de Investigaciones Agronómicas, Universidad de Costa Rica. San José, Costa Rica.

\begin{abstract}
Chemical properties and classification of soils cultivated with banana in the Caribbean lowlands of Costa Rica. Soils from 11 watersheds planted with banana in the aluvial valleys of the Caribbean zone of Costa Rica were chemically characterized and classified. Two major soil areas were identified based on their morphological and chemical properties and taxonomic criteria. One of the areas consisted mainly of Inceptisols formed from fluvio-volcanic materials with andic properties (Andic Eutrudepts, Aquandic Endoaquepts), dystric Inceptisols (Fluventic Dystrudepts) and Andisols (Typic Hapludands), most with good drainage, medium textures (sandy loam, loam, silty loam), sum of bases 10 to $19 \mathrm{cmol}^{-1} \mathrm{l}^{-1}$ CEC 13 to $28 \mathrm{cmol}^{\mathrm{l}^{-1}}$ and clays with permanent charge. The adjacent zone consisted mainly of Inceptisols formed from aluvial sedimentary materials deposited by frequent river floods (Fluventic Endoaquepts, Dystric Fluventic Eutrudepts, Fluvaquentic Eutrudepts, Fluvaquentic Endoaquepts), with a sum of bases

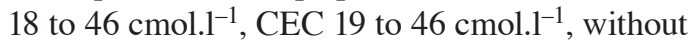
Andic properties, textures fine to medium (clay loam, silty clay loam, clay, silty clay) and in some case with poor drainage (Endoaquepts, Epiaquepts, Fluvaquentic). Geographically, the

\footnotetext{
*** CORBANA (Corporación Bananera Nacional). Guápiles, Costa Rica.

**** Escuela de Geología, Universidad de Costa Rica. San José, Costa Rica.
} 
limosa, arcillosa, arcillosa limosa) y con problemas de drenajes en algunos casos (Endoaquepts, Epiaquepts, Fluvaquentic). Geográficamente, estas zonas fueron separadas por la subcuenca formada por los ríos Vueltas-Silencio-Parismina, de forma que hacia el noroeste se encuentran suelos con influencia volcánica y fertilidad media, y hacia el sureste suelos sin influencia volcánica y de fertilidad alta.

\section{INTRODUCCIÓN}

El cultivo de banano, es una de las actividades agrícolas más importantes para la economía de Costa Rica, tanto por la generación de divisas (US\$ 674 mill., año 2008), como por la generación de empleos (140 000, año 2008). Las áreas de cultivo se encuentran principalmente en las llanuras del Caribe y cubren alrededor de 40 255 hectáreas (CORBANA 2009).

La caracterización de suelos de las plantaciones bananeras en el Caribe se inicia a principios del siglo XX (Prescot 1918, Bennett 1926). Posteriormente, se hacen otros estudios de caracterización regional (Nuhn y Pérez 1967, Jiménez 1972) y otra serie de trabajos de caracterización de fincas para el programa de fomento bananero, exigidos por el Sistema Bancario Nacional y generados durante el período 1977 a 1992. La mayoría de los estudios realizados para el programa de fomento bananero llevados a cabo por profesionales nacionales y de carácter privado son de difícil acceso; sin embargo están incluidos en el Atlas de la zona Atlántica Norte de Costa Rica resumido y digitado por Stoorvoguel y Eppink (1995). Los trabajos más recientes relacionados con el tema en la zona Caribe norte son los de Nieuwenhuyse (1996) en los cuales se describen las principales características ambientales de la región. Según Nieuwenhuyse (1996) en la zona de estudio se distinguen geomorfológicamente llanuras aluviales del Holoceno y planicies aluviales disectadas del Pleistoceno,
2 zones were separated by the watersheds of Vueltas-Silencio-Parismina Rivers; the northwest area is highly influenced by volcanic ash deposits and has its medium fertility characteristics, while the southeast area is not influenced by volcanic ejecta and has high fertility characteristics.

aunque en su mayoría son de origen muy reciente (100-5000 años), incluyen tanto suelos minerales (dominantes) como orgánicos, sometidos a tectonismo activo (Cohen et al. 1986, Nieuwenhuyse y Kroonenberg 1994). En la zona Caribe sureste, Luijckx y Zunnenberg (1992) distinguen 4 unidades fisiográficas en los sectores planos, a saber: Llanura Costera, Llanuras aluviales de los ríos grandes, Abanicos Aluviales y Cuencas de Deposición Interna, con descripción en la Llanura Costera, Typic Tropopsamments, Typic Eutropepts, Hydric y Fluvaquentic Tropofibrists y Typic Hydraquents, en la Llanura Aluvial de los ríos grandes Fluventic Eutropepts, en los Abanicos Aluviales Andic y Fluvaquentic Eutropepts, Fluvaquentic Dystropepts, Typic y Epiaquic Tropoudults y en la Cuenca de deposición Interna Typic Eutropepts y Typic Dystropepts.

La zona Caribe del país se divide en 2 grandes regiones geológicas (Dóndoli et al. 1968), de manera que los suelos del sector Caribe noroeste se desarrollan sobre coladas de lava, lahares, rocas piroclásticas, ignimbritas, rocas andesíticas, basaltos, cenizas volcánicas del cuaternario y pocas rocas sedimentarias. El Caribe sureste tiene suelos derivados de materiales provenientes de depósitos marino clásticos finos, calizas locales y aluviones de origen sedimentario. Las calizas además, son la causa de la gran inestabilidad de los terrenos de la parte alta de estas cuencas (Mora 2007).

Lara (1970) y Jiménez (1972) utilizan el río Reventazón como límite geográfico entre los 
2 sectores; más aún Jiménez considera que los suelos del noroeste son de fertilidad baja y los del sureste de fertilidad alta. Las llanuras aluviales del Caribe (noroeste y sureste) incluyen las principales zonas de producción de banano, distribuidas en 11 cuencas hidrográficas conformadas por ríos que nacen en las cordilleras Volcánicas, Central y Talamanca.

La edad de los suelos de la región noroeste de la zona Atlántica de Costa Rica se estima en varios trabajos, 2 de ellos emplean ${ }^{14} \mathrm{C}$ (Cohen et al. 1986, Dechesne 1994) y los otros el contenido total de elementos, a partir de que el Ti es inmóvil (Nieuwenhuyse et al. 1994a,b, Nieuwenhuyse y van Bremen 1997). Cohen et al. (1986), al estudiar los depósitos de turba en la cuenca baja del río Parismina, mencionan la presencia de estratos de sedimentos de origen volcánico (cenizas) alternándose con estratos de turba; los autores datan la parte superior del segundo estrato de turba a $270 \mathrm{~cm}$ de profundidad en 2330 años $\left({ }^{14} \mathrm{C}\right)$ y la parte superior del tercer estrato orgánico a 430 $\mathrm{cm}$ de profundidad en 3370 años $\left({ }^{14} \mathrm{C}\right)$. Dechesne (1994), al estudiar la deposición de sedimentos aluviales en la parte media de las cuencas de los ríos Tortuguero y Jiménez, estimaron la edad de la base de los sedimentos en canales de drenaje a $200-300 \mathrm{~cm}$ de profundidad entre $2200 \pm 75$ y $2975 \pm 110$ años $\left({ }^{14} \mathrm{C}\right)$, y correlacionaron la edad de estos sedimentos con períodos eruptivos del volcán Turrialba. De estos trabajos se deduce que: 1) los suelos en la superficie actual son mucho más jóvenes que los 2000-3000 años mencionados anteriormente, que 2) la deposición de materiales está íntimamente ligada al vulcanismo activo de la Cordillera Volcánica Central y 3) que pueden ocurrir fenómenos devastadores cada 1000 años, lo que permite enterrar gran cantidad de restos vegetales a partir de los cuales pueden formarse suelos orgánicos.

Los trabajos de Nieuwenhuyse et al. (1994a,b) y Nieuwenhuyse y van Bremen (1997) se concentran en la génesis de suelos derivados de ceniza volcánica andesítica en frentes de playa y la cabecera de la llanura aluvial de la zona Atlántica; los autores concluyen que en la extensa llanura aluvial se presentan suelos de textura variable de menos de 2000-5000 años (Holoceno) y suelos arcillosos de más de 50000 años en terrazas del Pleistoceno. Los suelos arenosos de los frentes de playa se consideran con menos de 500-2000 años de edad.

De acuerdo con Vargas (2004) la región Caribe de América Central presenta lluvias durante todo el año y no existe en ella una estación seca definida; en esta vertiente, la distribución de lluvias presenta 2 máximas (julio y diciembre) y 2 mínimas. En las llanuras del Caribe de Costa Rica llueven de 3915 (Puerto Limón) a 5420 (Barra del Colorado) mm.año ${ }^{-1}$, al ser el sector noroeste el más lluvioso; por esta razón los regímenes de humedad del suelo que se presentan son considerados como údico, perúdico y ácuico (Soil Survey Staff 2006). Como la temperatura media de toda la región es superior a $\operatorname{los} 24^{\circ} \mathrm{C}$ y la mayoría de las tierras están por debajo de los $300 \mathrm{msnm}$, el régimen de temperatura del suelo se considera como isohipertérmico (Soil Survey Staff 2006).

La precipitación media del sector noroeste es de 4282 mm.año ${ }^{-1}$ y la del sector sureste 3396 mm.año ${ }^{-1}$; donde Sixaola es el sector con menor precipitación (3058 mm.año-1) y Sarapiquí el sector de mayor precipitación $\left(4789 \mathrm{~mm} . \mathrm{mño}^{-1}\right)$. La temperatura máxima oscila entre 29,5 y $31,4^{\circ} \mathrm{C}$; la media entre 24,5 y $26,0^{\circ} \mathrm{C}$ y la mínima entre 20,3 y $21,9^{\circ} \mathrm{C}$. En el Cuadro 1 y la Figura 1 se detallan los datos de temperatura y precipitación de estaciones meteorológicas ubicadas en el área de estudio.

De acuerdo con Holdridge (1996) las llanuras del Caribe se ubican en las zonas de vida Bosque Muy Húmedo Tropical en el noroeste y central y Bosque Húmedo Tropical en el sureste. Sin embargo, aunque originalmente la zona estuvo cubierta de densos bosques, en la actualidad el sector comprendido entre los ríos San Juan (límite noreste de zona) y Matina, colonizado hace 300 años, tiene otro tipo de cobertura, mientras que del río Matina hacia el sureste la zona fue colonizada hace 140 años, cuando se construyó el ferrocarril (Nieuwenhuyse 1996). Alvarado 
Cuadro 1. Datos climatológicos de 10 estaciones meteorológicas ubicadas en las llanuras del Caribe de Costa Rica.

\begin{tabular}{|c|c|c|c|c|c|c|}
\hline Estación & Ubicación política & Años & $\begin{array}{l}\text { Precipitación } \\
\text { media } \\
\left(\mathrm{mm} \cdot \mathrm{año}{ }^{-1}\right)\end{array}$ & $\begin{array}{c}\text { Temp. Media } \\
\left({ }^{\circ} \mathrm{C}\right)\end{array}$ & $\begin{array}{c}\text { Temp. } \\
\text { Máx. }\left({ }^{\circ} \mathrm{C}\right)\end{array}$ & $\begin{array}{c}\text { Temp. } \\
\text { Mín. }\left({ }^{\circ} \mathrm{C}\right)\end{array}$ \\
\hline \multicolumn{7}{|c|}{ Región noroeste } \\
\hline Corbana & Pococí, La Rita de Guápiles & $1989-2005$ & 4104,7 & 24,5 & 30,5 & 20,3 \\
\hline Mola 1 & Pococí, Duacarí & $1972-2004$ & 3948,5 & 25,1 & 30,5 & 21,5 \\
\hline Finca 1 & Sarapiquí, Horquetas, Río Frío & $2000-2005$ & 4442,2 & nd & 30,5 & 20,4 \\
\hline Zurquí 1 & Sarapiquí, Horquetas, Río Frío & $2001-2005$ & 4787,5 & nd & 29,5 & 20,4 \\
\hline La Selva & Sarapiquí, Horquetas & $1992-2004$ & 4127,2 & nd & 30,9 & 21,5 \\
\hline \multicolumn{7}{|c|}{ Región sureste } \\
\hline Daytonia & Talamanca, Sixaola, Daytonia & $1996-2003$ & 3157,0 & 26,0 & $31,0^{*}$ & $20,5^{*}$ \\
\hline Fortuna & Limón, Matama, Fortuna & $2000-2005$ & 3058,8 & nd & 31,4 & 21,1 \\
\hline Los Ríos & Limón, Rio Blanco, Bananito Sur & $2003-2005$ & 3454,0 & nd & 30,9 & 21,9 \\
\hline Corbana & 28 Millas, Matina, Bataan & $1989-2005$ & 3586,0 & 24,9 & 30,6 & 20,8 \\
\hline Carmen 1 & Siquirres, Siquirres, El Carmen & $1972-2004$ & 3785,1 & 25,3 & 30,5 & 21,4 \\
\hline
\end{tabular}

* Los datos de $\mathrm{t}$ max y $\mathrm{t}$ min corresponden a valores promedios para el año 2005.

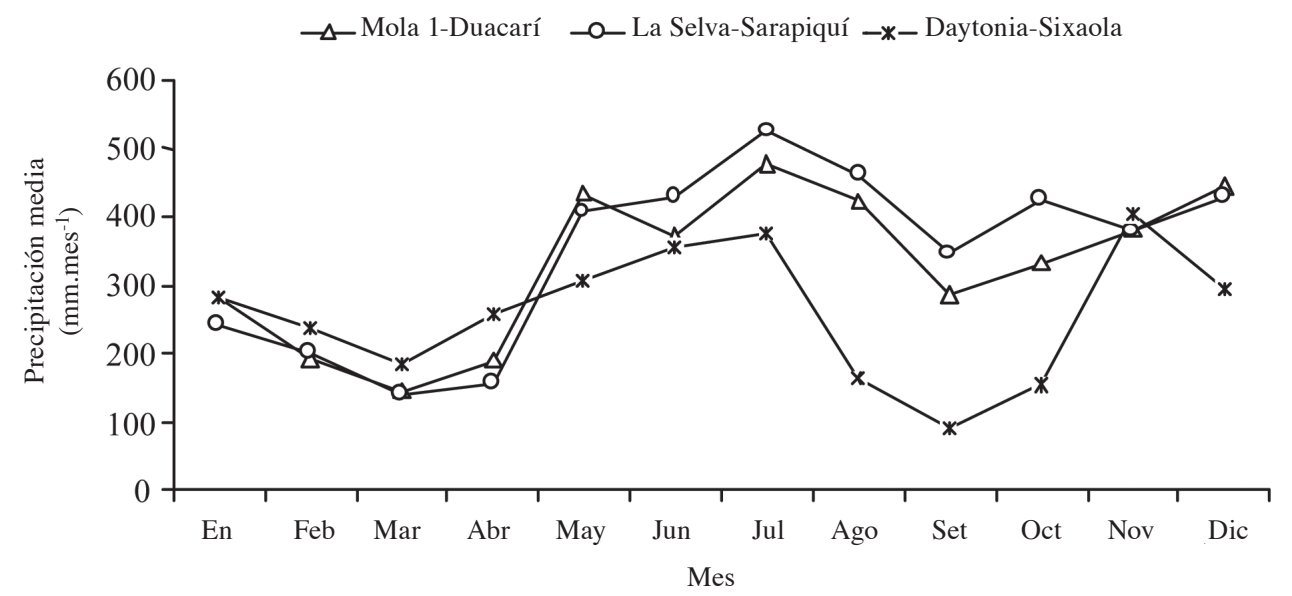

Fig. 1. Distribución de precipitación en las estaciones Mola 1, La Selva y Daytonia.

(2006) menciona que en éstas zonas de vida del piso tropical de Costa Rica es donde se produce la mayor cantidad de biomasa debido a la mayor pluviosidad y otros autores documentan los cambios que sufre esta fracción con cambios en la vegetación original (Vargas y Flores 1995, van Dam et al. 1997, Guggenberger y Zech 1999, Powers
2001 2004, Powers y Schlesinger 2002a,b, Russell et al. 2004).

En vista de que el cultivo de banano es de suma relevancia para la economía del país y que es poco lo que se ha publicado sobre las propiedades químicas, físicas y morfológicas de los suelos de esta región, el objetivo de esta investigación es 
realizar una caracterización del agro ecosistema y una caracterización de los suelos en áreas cultivadas de banano en las 11 cuencas que conforman las llanuras aluviales del Caribe de Costa Rica.

\section{MATERIALES Y MÉTODOS}

\section{Descripción del área de estudio}

La investigación se realizó en fincas bananeras ubicadas en las llanuras aluviales de las cuencas hidrográficas de los ríos Sixaola, Estrella, Moín, Banano, Matina, Madre de Dios, Pacuare y Reventazón que pertenecen a las llanuras del Caribe Sureste; y Tortuguero, Chirripó Caribe y Sarapiquí que pertenecen a las llanuras del Caribe Noroeste de Costa Rica. En la zona bananera Atlántica existen alrededor de 150 fincas con una área de 40751 ha distribuidas en las cuencas de los ríos Sixaola (2288 ha), Estrella (1652 ha), Banano (916 ha), Moín (2665 ha), Matina (5954 ha), Madre de Dios (5886 ha), Pacuare (1611 ha), Reventazón (4087 ha), Tortuguero (9852 ha), Chirripó Caribe (2618 ha) y Sarapiquí (3138 ha). En la Figura 2 se detalla el área de influencia de cada cuenca y los sitios estudiados.

A. Depósitos aluviales y coluviales del cuaternari

B. Rocas sedimentarias del Mesozoico-Cenozoi

C. Rocas volcánicas de cuaternario. (Según Tournon y Alvarado 1995).

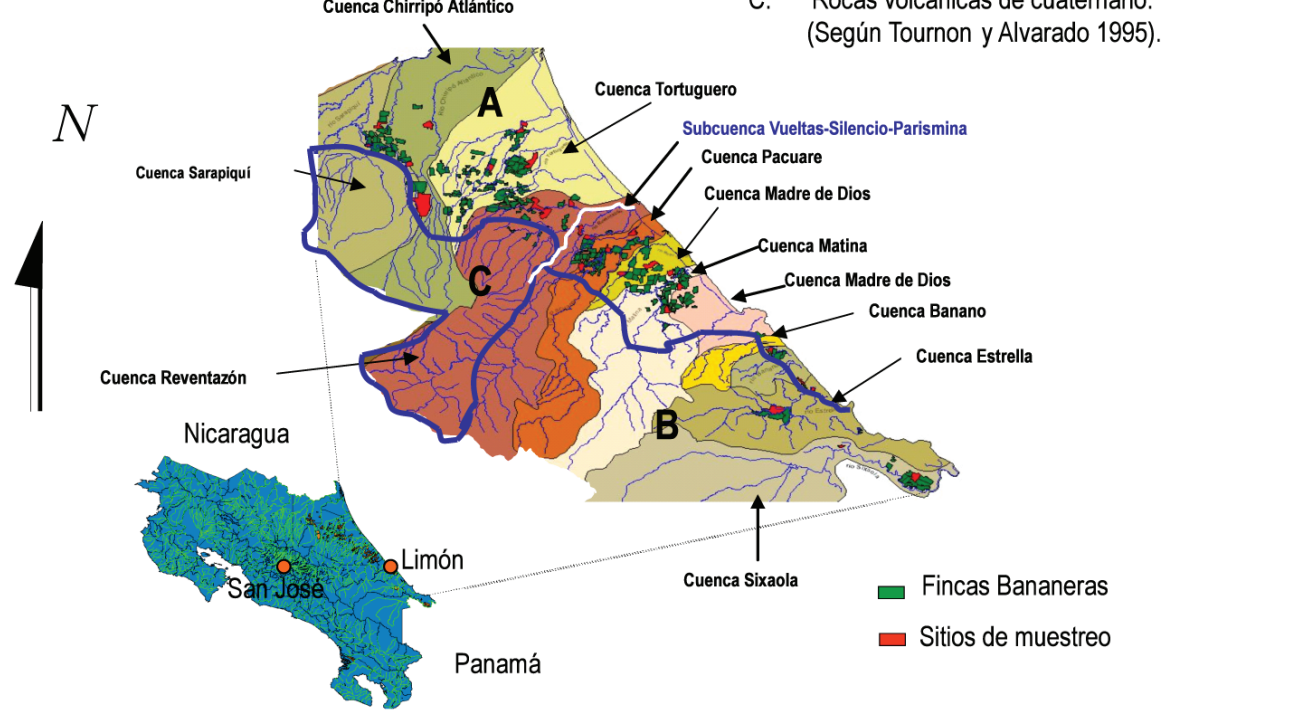

Fig. 2. Localización geográfica del área de estudio, geología y distribución de las fincas bananeras por cuenca.

\section{Muestreo y análisis de suelos}

Para la caracterización taxonómica y química de los suelos se seleccionaron fincas con características de suelos y manejo representativas cada una de las cuencas estudiadas. Se utilizó como criterio de separación de unidades de muestreo el área de influencia de cada cuenca; aunque también se utilizó como referencia estudios de clasificación de suelos de la Región Atlántica de varios autores, resumidos y digitados por Stoorvoguel y Eppink (1995). En total se seleccionaron 24 fincas en toda la región bananera. Se seleccionó una finca en cada una de las cuencas de los ríos Estrella, Bananito y Moín; 2 fincas por cuenca en Sixaola, Matina, Madre de Dios y Sarapiquí; 3 fincas por cuenca en Pacuare, Reventazón y Chirripó y 4 fincas en la cuenca del Tortuguero (Cuadro 2). 
Una vez seleccionada la finca se ubicó un suelo típico (geomorfología y manejo de cultivo) del área de estudio y se abrió una calicata para describir y hacer la descripción del perfil y la toma de muestras de suelo por horizonte genético según lineamientos de National Soil Survey Center (Versión 2 2002). La clasificación taxonómica hasta nivel de subgrupo se realizó por medio de la clave para taxonomía de suelos del Soil Survey Staff (2006). El pH en $\mathrm{H}_{2} \mathrm{O}, \mathrm{KCl}$ y $\mathrm{NaF}$, se midieron todos en relación 1:2,5, y la acidez intercambiable en $\mathrm{KCl} 1 \mathrm{~N}$ se determinó según metodología Soil Survey Staff (2006). La
CIC se determinó con $\mathrm{NH}_{4} \mathrm{OAc} 1 \mathrm{~N}$ de acuerdo con el método de Chapman (1965); el K, Ca y Mg intercambiables se extrajeron con una solución de $\mathrm{NH}_{4} \mathrm{OAc} 1 \mathrm{~N}$ y se determinaron por medio de espectrofotometría con un plasma inductivamente acoplado (E-ICP); la retención de fosfatos se determinó según el método de Olsen y Sommers (1982) y el aluminio e hierro asociados con materiales amorfos fue extraído con oxalato de amonio y medido con E-ICP. La materia orgánica (MO) se estimó por el método de Walkey y Black, descrito por Nelson y Sommers (1982).

Cuadro 2. Ubicación geográfica de fincas y sitios estudiados en cuencas de las llanuras del Caribe noroeste y sureste.

\begin{tabular}{|c|c|c|c|c|}
\hline Cuenca & Nombre de finca & Código & $\begin{array}{l}\text { Ubicación geográfica } \\
\text { (Provincia/Cantón/Distrito/Poblado) }\end{array}$ & Coordenadas \\
\hline \multicolumn{5}{|c|}{ Caribe noroeste } \\
\hline Reventazón & Banasol-ACON & RE-15 & Limón, Guácimo, Rio Jiménez & $10^{\circ} 16^{\prime} 14,2^{\prime \prime} \mathrm{N} ; 83^{\circ} 34^{\prime} 48,8^{\prime \prime} \mathrm{W}$ \\
\hline \multirow{4}{*}{ Tortuguero } & Lomas de Sierpe & TO-16 & Limón, Pococí, Roxana & $10^{\circ} 24^{\prime} 57,6^{\prime \prime} \mathrm{N} ; 83^{\circ} 35^{\prime} 30,8^{\prime \prime} \mathrm{W}$ \\
\hline & Cahuita-SIELSA & TO-17 & Limón, Pococí, Cariari & $10^{\circ} 31^{\prime} 51,8^{\prime \prime} \mathrm{N} ; 83^{\circ} 57^{\prime} 27,2^{\prime}{ }^{\prime} \mathrm{W}$ \\
\hline & Taciafa & TO-18 & Limón, Pococí, Cariari & $10^{\circ} 25^{\prime} 18^{\prime \prime} \mathrm{N} ; 83^{\circ} 41^{\prime} 39,3^{\prime \prime} \mathrm{W}$ \\
\hline & La Rita- CORBANA & TO-19 & Limón, Pococí, Pococí, La Rita & $10^{\circ} 15^{\prime} 54^{\prime \prime} \mathrm{N} ; 8^{\circ} 46^{\prime} 26^{\prime \prime} \mathrm{W}$ \\
\hline \multirow{3}{*}{ Chirripó } & $\begin{array}{l}\text { Inv. Santa María del } \\
\text { Monte }\end{array}$ & $\mathrm{CH}-20$ & Heredia, Sarapiquí, Puerto Viejo & $10^{\circ} 25^{\prime} 48^{\prime \prime} \mathrm{N} ; 83^{\circ} 54^{\prime} 36^{\prime \prime} \mathrm{W}$ \\
\hline & Chiras-SIELSA & $\mathrm{CH}-21$ & Heredia, Sarapiquí, Puerto Viejo & $10^{\circ} 33^{\prime} 07,1^{\prime \prime} \mathrm{N} ; 83^{\circ} 57^{\prime} 53,4^{\prime \prime} \mathrm{W}$ \\
\hline & Victoria & $\mathrm{CH}-22$ & Heredia, Sarapiquí, Puerto Viejo & $10^{\circ} 32^{\prime} 06,8^{\prime \prime} \mathrm{N} ; 83^{\circ} 51^{\prime} 38,5^{\prime \prime} \mathrm{W}$ \\
\hline \multirow{2}{*}{ Sarapiquí } & Inversiones Orlich & SA-23 & Heredia, Sarapiquí, Horquetas & $10^{\circ} 28^{\prime} 48^{\prime \prime} \mathrm{N} ; 8^{\circ} 00^{\prime} 36^{\prime \prime} \mathrm{W}$ \\
\hline & Rio Frío-DOLE & SA-24 & Heredia, Sarapiquí, Horquetas, Rio Frío & $10^{\circ} 17^{\prime} 37,5^{\prime \prime} \mathrm{N} ; 83^{\circ} 52^{\prime} 10,8^{\prime \prime} \mathrm{W}$ \\
\hline \multicolumn{5}{|c|}{ Caribe sureste } \\
\hline \multirow{2}{*}{ Sixaola } & Daytonia- CORBANA & SI-1 & Limón, Talamanca, Sixaola, Daytonia & $9^{\circ} 31^{\prime} 48^{\prime \prime} \mathrm{N} ; 82^{\circ} 39^{\prime} 36^{\prime \prime} \mathrm{W}$ \\
\hline & Platanera Rio Sixaola & SI-2 & Limón, Talamanca, Sixaola, Bratsi & 9॰37’12”' N; 8250’24”' W \\
\hline Estrella & Fortuna-DOLE & ES-3 & Limón, Limón, Matama, Fortuna & $9^{\circ} 45^{\prime} 9,7^{\prime \prime} \mathrm{N} ; 83^{\circ} 00^{\prime} 21,9^{\prime \prime} \mathrm{W}$ \\
\hline Bananita & Los Ríos-DOLE & BA-4 & Limón, Limón, Rio Blanco, Bananito Sur & $9^{\circ} 53^{\prime} 29,3^{\prime \prime} \mathrm{N} ; 83^{\circ} 00^{\prime} 08,2^{\prime \prime} \mathrm{W}$ \\
\hline Moín & Montebello-ACON & MO-5 & Limón, Matina, Carrandí & $10^{\circ} 04^{\prime} 29,8^{\prime \prime} \mathrm{N} ; 83^{\circ} 14^{\prime} 20^{\prime \prime} \mathrm{W}$ \\
\hline \multirow{2}{*}{ Matina } & Acumi & MA-6 & Limón, Matina, Matina & $10^{\circ} 06^{\prime} 52,9^{\prime \prime} \mathrm{N} ; 83^{\circ} 15^{\prime} 22,3^{\prime \prime} \mathrm{W}$ \\
\hline & Zent & MA-7 & Limón, Matina, Carrandí & $10^{\circ} 00^{\prime} 26^{\prime \prime} \mathrm{N} ; 83^{\circ} 15^{\prime} 54^{\prime \prime} \mathrm{W}$ \\
\hline \multirow{2}{*}{$\begin{array}{l}\text { Madre de } \\
\text { Dios }\end{array}$} & Guaria-UNIBAN & MD-8 & Limón, Matina, Bataan & $10^{\circ} 07^{\prime} 45,2^{\prime \prime} \mathrm{N} ; 83^{\circ} 18^{\prime} 55,6^{\prime \prime} \mathrm{W}$ \\
\hline & San Pablo-CORBANA & MD-9 & Limón, Siquirres, Pacuarito & $10^{\circ} 07^{\prime} 12^{\prime \prime} \mathrm{N} ; 83^{\circ} 22^{\prime} 48^{\prime \prime} \mathrm{W}$ \\
\hline \multirow{3}{*}{ Pacuare } & $\begin{array}{l}\text { Nueva } \\
\text { Esperanza-SUNISA }\end{array}$ & PA-10 & Limón, Siquirres, Siquirres & $10^{\circ} 13^{\prime} 28^{\prime \prime} \mathrm{N} ; 83^{\circ} 21^{\prime} 53^{\prime \prime} \mathrm{W}$ \\
\hline & Imperio- BANDECO & PA-11 & Limón, Siquirres, Siquirres & $10^{\circ} 10^{\prime} 48^{\prime \prime} \mathrm{N} ; 8^{\circ} 25^{\prime} 12^{\prime \prime} \mathrm{W}$ \\
\hline & Siquirres & PA-12 & Limón, Siquirres, Siquirres & $10^{\circ} 07^{\prime} 48^{\prime \prime} \mathrm{N} ; 83^{\circ} 28^{\prime} 48^{\prime \prime} \mathrm{W}$ \\
\hline \multirow{2}{*}{ Reventazón } & Carmen 1- BANDECO & RE-13 & Limón, Siquirres, Siquirres, El Carmen & $10^{\circ} 12^{\prime} 36^{\prime \prime} \mathrm{N} ; 83^{\circ} 28^{\prime} 47^{\prime \prime} \mathrm{W}$ \\
\hline & Catalina-SIELSA & RE-14 & Limón, Siquirres, El Cairo & $10^{\circ} 13^{\prime} 48^{\prime \prime} \mathrm{N} ; 83^{\circ} 28^{\prime} 40^{\prime \prime} \mathrm{W}$ \\
\hline
\end{tabular}

Agronomía Costarricense 34(2): 177-195. ISSN:0377-9424 / 2010 


\section{RESULTADOS Y DISCUSIÓN}

Los suelos estudiados permiten caracterizar las condiciones edáficas más representativas de las fincas bananeras, ubicadas en las cuencas de los ríos Chirripó Caribe, Sarapiquí, Tortuguero y Reventazón de las llanuras de Caribe noroeste y los ríos Pacuare, Madre de Dios, Matina, Moín, Banano, Estrella y Sixaola de las llanuras del Caribe sureste (Cuadro 3).

\section{Morfología}

Las principales características morfológicas de los suelos estudiados del noroeste están definidas por deposiciones coluvio-aluviales de origen volcánico; con secuencias de horizontes con cambios texturales abruptos, contenidos de materia orgánica que varían de forma irregular con la profundidad de los diferentes perfiles, presencia de lechos de río y desechos de plástico enterrados (localmente conocidos como bolsas, piola o pita). Así mismo, sus horizontes reflejan características heredadas de cenizas y otros materiales volcánicos, tales como baja densidad aparente, consistencia friable, texturas medias y colores oscuros.

En general estos suelos presentan un epipedón úmbrico sobre un endopedón cámbico con una secuencia normal de los horizontes Ap-Bw$\mathrm{Cg}$. El epipedón, normalmente está modificado por actividades humanas de manera que se reconoce como un horizonte Ap, de color pardo a pardo oscuro, con estructura en forma de bloques subangulares y un grosor promedio de $16 \mathrm{~cm}$, con mínimos de $10 \mathrm{~cm}$ y máximos de $40 \mathrm{~cm}$. Los horizontes subsuperficiales son de color pardo amarillento oscuro con moteos grises $(\mathrm{Bw})$ y gleización $(\mathrm{Bg}, \mathrm{Cg})$ con una gran variación en el grosor; con mínimo de $4 \mathrm{~cm}$ y máximo de $78 \mathrm{~cm}$. Los horizontes $\mathrm{Bw}$ denotan desarrollo estructural, escasa acumulación de arcillas, estructura en forma de bloques subangulares; los $\mathrm{Bg}$ y $\mathrm{Cg}$ normalmente carecen de estructura y si la poseen es de bloques de débil desarrollo. El proceso de gleización es causado por la presencia de un drenaje interno lento combinado con niveles freáticos altos, típicos de regiones aluviales con sistemas hídricos meándricos con empozamiento y saturación del suelo y adicionalmente mal funcionamiento del sistema de drenaje, por mal diseño o falta de mantenimiento. Se encontraron 2 horizontes enterrados $(\mathrm{Ab})$ producto de las frecuentes inundaciones (Andic Eutrudepts, perfiles 3 a 5) y 2 suelos con horizontes parcialmente compactados sin que presentaran restricción al crecimiento de raíces (Fluventic Endoaquepts, perfil 4; Fluvaquentic Eutrudepts, perfil 5).

La mayoría de los suelos presentaron condiciones aceptables para el crecimiento de las raíces, las cuales se observaron hasta $1,5 \mathrm{~m}$ de profundidad, distribuidas en su mayoría en los horizontes Ap y Bw, con excepción de algunos suelos con presencia de horizontes gleizados. En los suelos bien drenados la profundidad efectiva fue mayor a $90 \mathrm{~cm}$, valor definido como ideal para el crecimiento del banano por Jaramillo y Vásquez (1980), aunque otros autores (Araya y Blanco 2001, Araya et al. 1998) reportan que el sistema radical del banano se distribuye principalmente en los primeros $60 \mathrm{~cm}$ de profundidad. Los suelos mal drenados presentan algún grado de características redoximórficas y gleización (horizontes $\mathrm{Bg}$ ó $\mathrm{Cg}$ ), como es el caso del Fluventic Endoaquepts, perfil 4. En estos suelos es necesario diseñar sistemas de drenaje de alto costo, siempre y cuando posean características tales que los hagan aptos para el cultivo de banano.

En el sector sureste la morfología de los suelos está definida, al igual que en los suelos del noroeste, por su origen aluvial en un ambiente hidrológico meándrico de origen sedimentario marino, lo que causa condiciones de drenaje deficiente y clases texturales medias a finas, las cuales son producto de deposiciones muy lentas de sedimentos o no turbulentas, tal cual describen para la zona Luijckx y Zunnenberg (1992).

En general, estos suelos se distinguen por tener un epipedón úmbrico sobre un endopedón cámbico. Los horizontes Ap poseen estructura de bloques subangulares, con un grosor medio del horizonte de $13 \mathrm{~cm}$, con mínimos de $5 \mathrm{~cm}$ y máximos de $22 \mathrm{~cm}$. Los horizontes 


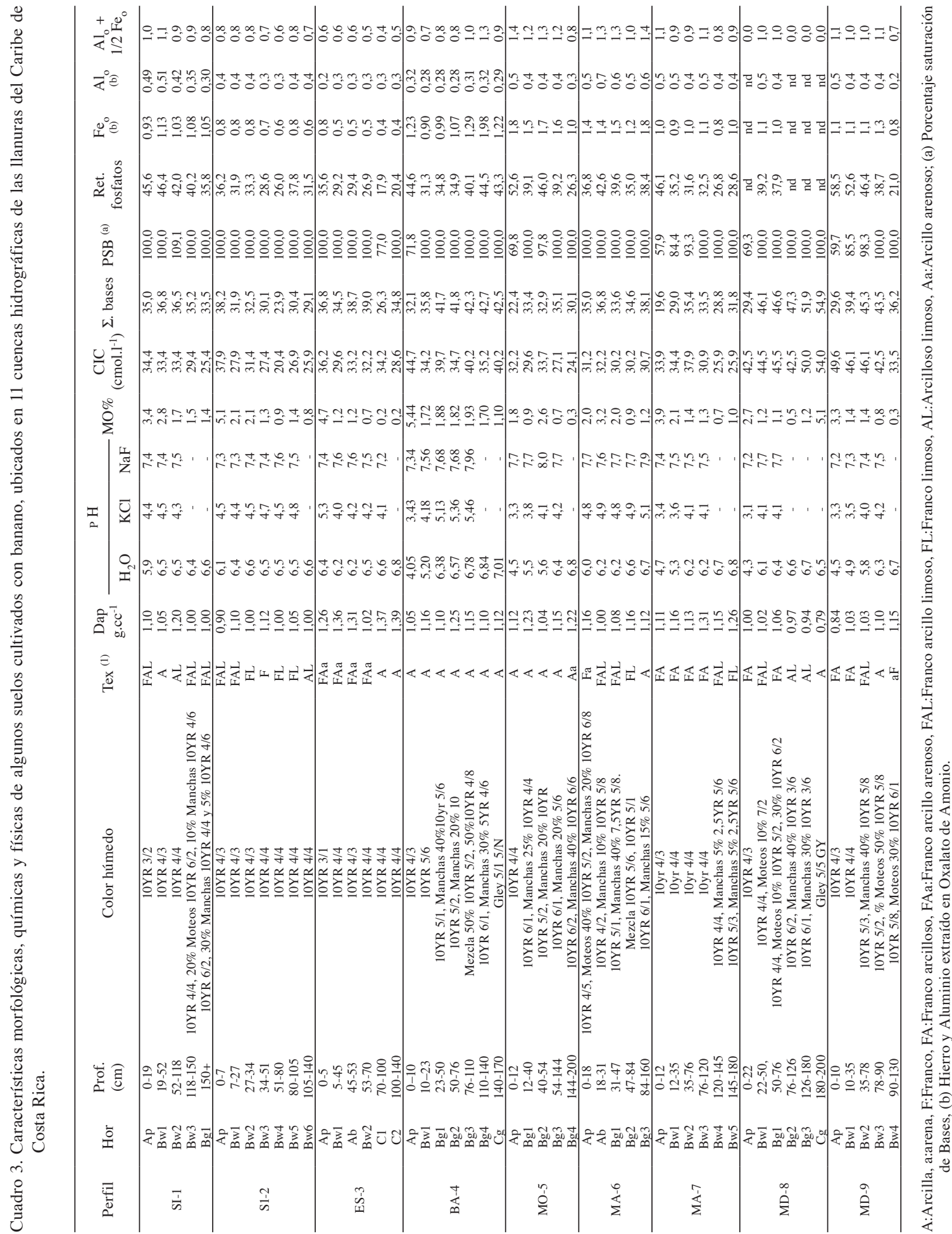




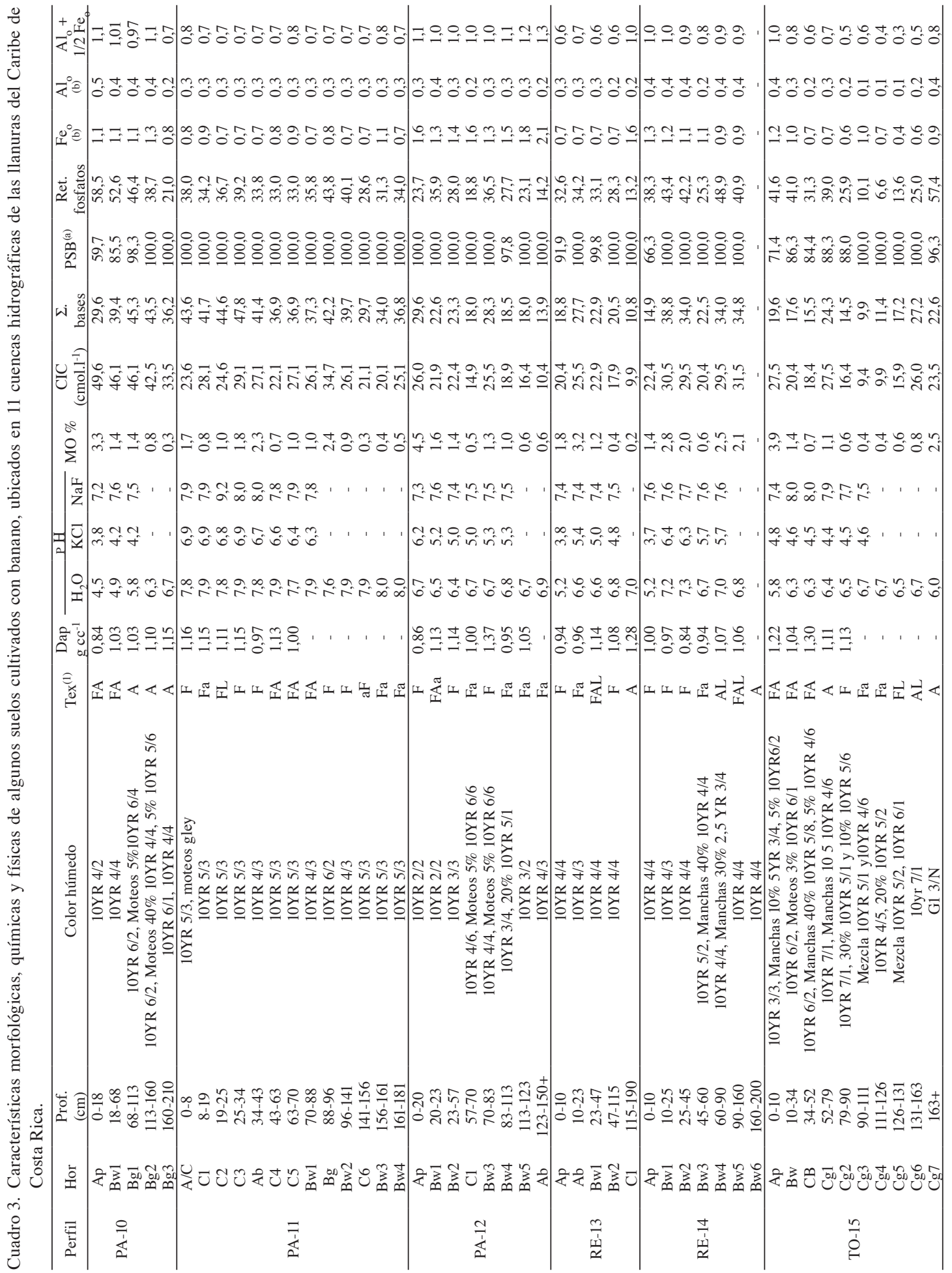




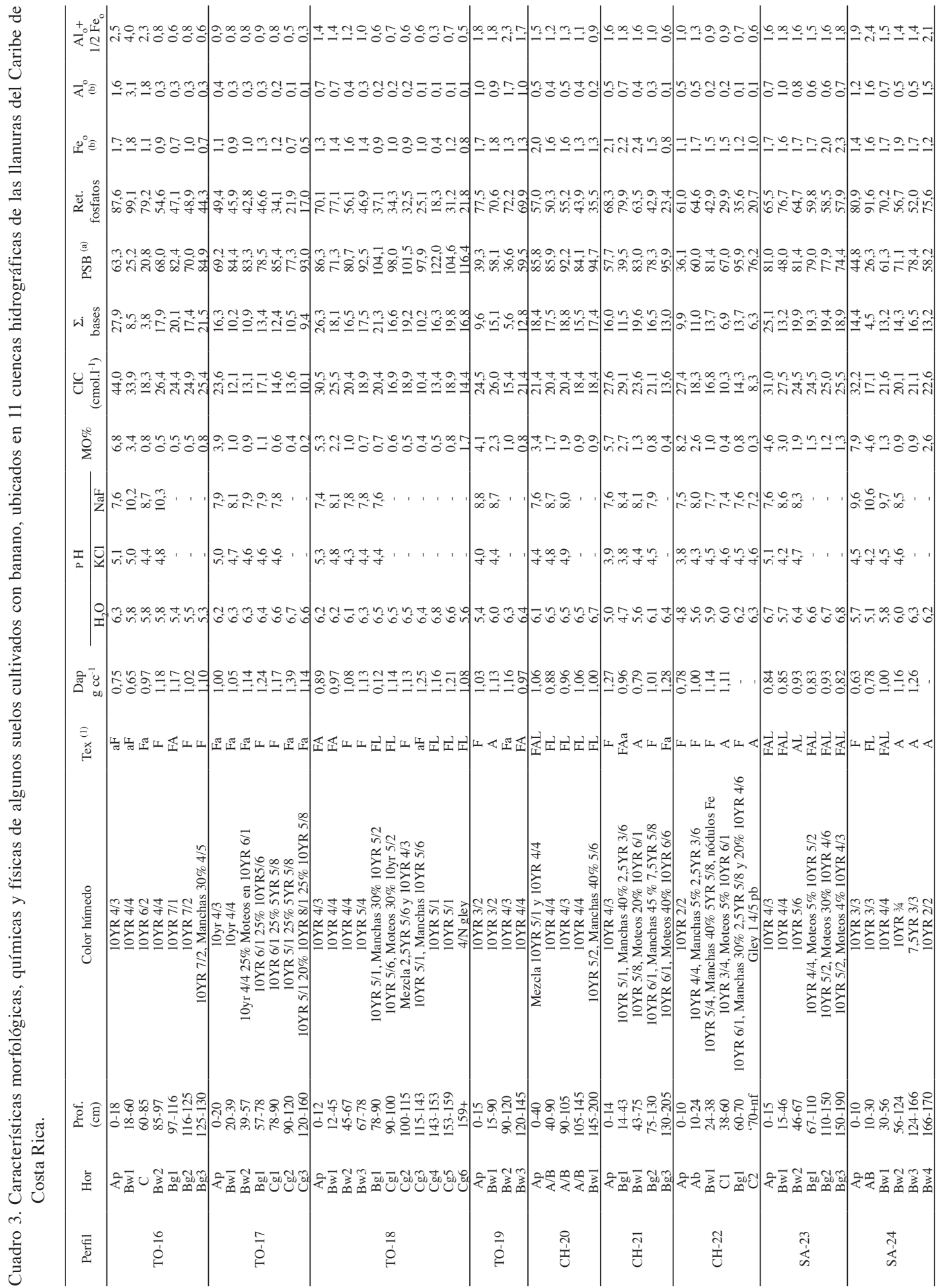


subsuperficiales son de color pardo amarillento oscuro con frecuentes moteos grises $(\mathrm{Bw}) \mathrm{y}$ gleización $(\mathrm{Bg}, \mathrm{Cg})$ con una fuerte variación en el grosor (con mínimo de $3 \mathrm{~cm}$ y máximo de $90 \mathrm{~cm}$ ). Los horizontes Bw denotan desarrollo estructural, escasa acumulación de arcillas, estructura de bloques subangulares; los $\mathrm{Bg}$ y $\mathrm{Cg}$ normalmente carecen de estructura y si la poseen es de bloques subangulares débiles. Los suelos con horizontes gleizados son más frecuentes que los de las llanuras de noroeste y a diferencia de esta zona, su recuperación es más compleja por la baja capacidad natural de drenaje. Se encontraron 4 horizontes $\mathrm{Ab}$ producto de las frecuentes inundaciones (Dystric Fluventic Eutrudepts, perfil 3; Fluventic Endoaquepts perfil 2; Typic Udifluvents, Fluvaquentic Eutrudepts, perfil 5) y 2 suelos con horizontes semi compactados, aunque no presentaron restricción al crecimiento de raíces (Dystric Fluventic Eutrudepts, perfiles 3 y 4). Las condiciones para el crecimiento de raíces fueron similares a las de las llanuras del noroeste, aunque la presencia de horizontes con condiciones redoximórficas y de gleización fue mayor que en los suelos del noroeste.

\section{Propiedades físicas}

Para suelos bananeros se considera que valores de densidad aparente (Da) mayores 1,30 Mg. $\mathrm{kg}^{-1}$ indican un grado de compactación moderada (Serrano 2002). En las llanuras del noroeste no se encontró compactación en los horizontes Ap (excepto en Fluventic Endoaquepts, perfil 4 y Fluvaquentic Eutrudepts, perfil 5) o en los endopedones. En esta zona se encuentran algunos suelos con características ándicas en algún horizonte del perfil (valores de Da menores a $0,90 \mathrm{Mg} \cdot \mathrm{kg}^{-1}$ ) y su variación con la profundidad es similar a la encontrada para Andisoles de Costa Rica por Alvarado y Forsythe (2005).

Los suelos de esta zona presentan en sus horizontes superficiales y subsuperficiales texturas que van desde arena franca hasta franco arcillosa. Los suelos con texturas más gruesas fueron el Fluvaquentic Eutrudepts, perfil 5, el Andic Eutrudepts, perfil 3 y el Typic Hapludans y los suelos con las texturas más finas fueron el Fluventic Dystrudepts y Fluventic Endoaquepts, perfil 4. Estas variaciones texturales se asocian con el fenómeno de deposición de los materiales aluviales, donde los suelos cercanos a los cauces son de textura gruesa y los más alejados son de textura más fina (Luijckx y Zunnenberg 1992).

En las llanuras del sureste, se encontró valores de Da mayores que en los suelos del noroeste; no se encontraron horizontes Ap compactados y solamente en los suelos Dystric Fluventic Eutrudepts, perfiles 3 y 4 y el suelo Fluvaquentic Eutrudepts, perfil 3 (asociada a la disminución de actividad biológica y efectos antropogénicos) se encontraron horizontes subsuperficiales compactados según el criterio de Serrano (2002). En esta zona, los suelos presentan

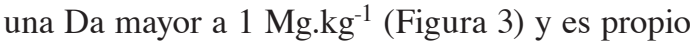
de los Inceptisoles de Costa Rica (Alvarado y Forsythe 2005). Las texturas franco arenosas a arcillosas en el Ap y franco arcilloso limosa a arcillosa en los horizontes subsuperficiales, reflejan la baja densidad del material parental calcáreo (Dóndoli et al. 1968), lo que facilita su meteorización, y la forma en que se depositan los materiales (Luijckx y Zunnenberg 1992); el suelo con texturas más gruesas fue el Typic Udifluvents, el cual corresponde a un sector del dique natural del río Pacuare y los suelos con las texturas más finas fueron el Fluvaquentic Epiaquepts, el Fluventic Endoaquepts, perfil 1 y el Fluvaquentic Eutrudepts, perfil 2, que se ubican en áreas bajas de las llanuras aluviales de los ríos Bananito y Pacuare. La predominancia de texturas más gruesas y densidad aparente más bajas provocan las mejores condiciones de drenaje de los suelos ubicados en la zona noroeste, en la que se ubican las cuencas de los ríos Chirripó, Sarapiquí, Tortuguero. Algunos suelos de textura gruesa de esta zona como resultado de su anisotropía textural, presentan un drenaje excesivo y no se consideran adecuados para el cultivo de banano debido a que su fertilidad natural es baja. 


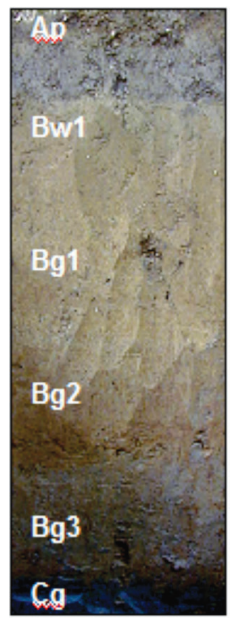

Fluvaquentic Eutrudepts

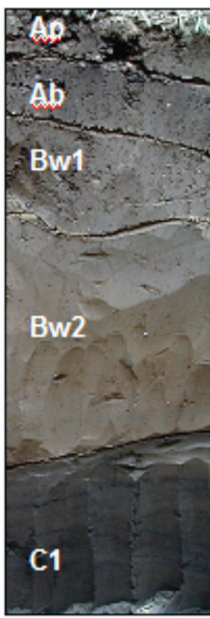

Distric Fluventic Eutrudepts

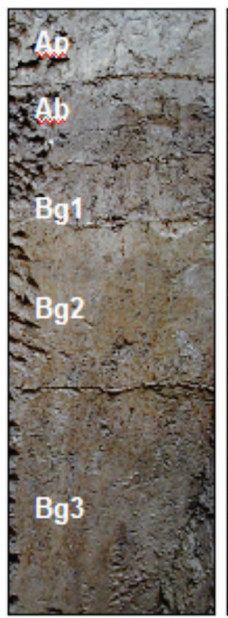

Fluventic

Endoaquepts

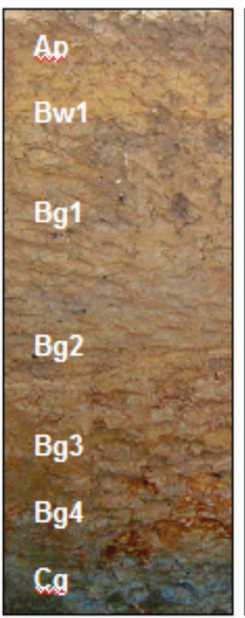

Fluvaquentic Epiaquepts

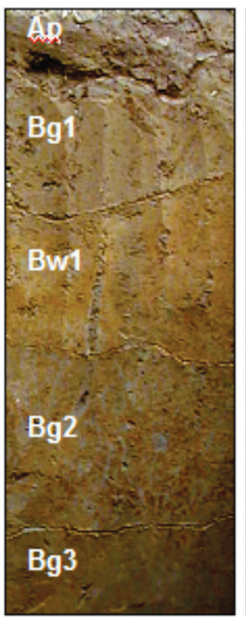

Aquandic

Endoaquepts

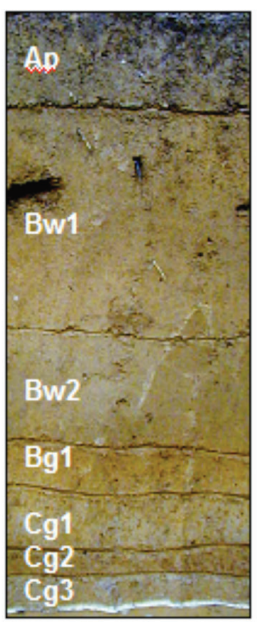

Typic Hapludands

Fig. 3. Perfiles típicos predominantes en suelos bananeros de las llanuras del Caribe de Costa Rica.

\section{Propiedades químicas}

Las características químicas de los perfiles se presentan en los Cuadros 3 y 4 . Los valores de $\mathrm{pH}\left(\mathrm{H}_{2} \mathrm{O}\right)$ fluctuaron entre 4,1 y 7,9 para ambas zonas, con un promedio de 6,3 y 6,1 en los suelos del sureste y el noroeste respectivamente. Los valores de $\mathrm{pH}\left(\mathrm{H}_{2} \mathrm{O}\right)$ fueron similares en ambas zonas, a pesar de las diferencias en material parental; lo cual podría ser causa de la elevada pluviosidad que tiende a lavar bases en forma permanente, a la elevada frecuencia de inundaciones de la región lo que causa deposición de materiales nuevos en forma constante (Nieuwenhuyse 1996) y a las prácticas de fertilización de las plantaciones, que tienden a uniformizar los valores. Sin embargo, cuando se observa la variación del $\mathrm{pH}$ en la banda de fertilización de plantaciones bananeras, se encuentra que en el suelo la acidificación ocurre a corto plazo y en el subsuelo a largo plazo (Serrano 2005). Los valores promedios de $\mathrm{pH}$ $\left(\mathrm{H}_{2} \mathrm{O}\right)$ encontrados son mayores a los reportados por López y Solís (1991) para suelos del sureste y noroeste, lo cual se debe a que el muestreo realizado por estos, fue a una profundidad de $0-30 \mathrm{~cm}$, lo que reflejo más la acidificación mencionada por Serrano (2005). El Typic Udifluvents fue el suelo con mayor $\mathrm{pH}$, único que presentó reacción al $\mathrm{HCl} 10 \%$, lo que evidencia la presencia de carbonatos libres. Los suelos con los menores $\mathrm{pH}$ $\left(\mathrm{H}_{2} \mathrm{O}\right)$ fueron el Fluventic Endoaquepts, perfil 3 y Fluvaquentic Eutrudepts perfil 2 de origen sedimentario marino y el Typic Hapludands y el Aquandic Endoaquepts de origen volcánico.

El pH (NaF) fue mayor a 8,2 solamente en algunos suelos del noroeste, lo cual supone la presencia de minerales de rango corto de cristalización, producto de la meteorización de piroclastos (Soil Suvey Staff 2006). Los suelos donde se encontraron algunos horizontes con $\mathrm{pH}$ $(\mathrm{NaF})$ mayor a 8,2 fueron el Typic Hapludands, el Fluventic Dystrudepts, los Andic Eutrudepts, perfiles 2-4-5 y el Aquandic Endoaquepts. En el horizonte $\mathrm{C} 1$ del Typic Udifluvents se encontró un $\mathrm{pH}$ mayor de 8,2 sin embargo por presentar dicho suelo carbonatos libres esa prueba no se considera relevante para indicar la presencia de minerales de rango corto de cristalización.

Los valores de capacidad de intercambio catiónico (CIC) de toda la región se consideran altos $\left(>20 \mathrm{cmol}^{-1} \mathrm{l}^{-1}\right)$, con un valor promedio para ambas zonas de $26,6 \mathrm{cmol}^{\mathrm{I}^{-1}}$, con un mínimo 
Cuadro 4. Valores promedio ${ }^{1}$ de características químicas de suelos cultivados con banano ubicados en 11 cuencas hidrográficas de las llanuras del Caribe de Costa Rica.

\begin{tabular}{|c|c|c|c|c|c|c|c|c|c|}
\hline \multirow[b]{2}{*}{ Suelo } & \multirow[b]{2}{*}{ Cuenca } & \multicolumn{3}{|c|}{$\mathrm{pH}$} & \multirow{2}{*}{$\begin{array}{c}\mathrm{MO} \\
\%\end{array}$} & \multirow{2}{*}{$\begin{array}{c}\text { CIC } \\
\left.\left(\mathrm{cmol}^{-1}\right)^{-1}\right)\end{array}$} & \multirow{2}{*}{$\begin{array}{c}\Sigma \\
\text { Bases }\end{array}$} & \multirow{2}{*}{$\begin{array}{l}\text { SB } \\
(\%)\end{array}$} & \multirow{2}{*}{$\begin{array}{c}\text { Ret. } \\
\text { Fosfatos } \\
(\%)\end{array}$} \\
\hline & & $\mathrm{H}_{2} \mathrm{O}$ & $\mathrm{KCl}$ & $\mathrm{NaF}$ & & & & & \\
\hline \multicolumn{10}{|c|}{ Caribe sureste } \\
\hline SI-1 & Sixaola & 6,4 & 4,4 & 7,4 & 2,2 & 31,2 & 35,4 & 100,0 & 42,0 \\
\hline SI-2 & Sixaola & 6,5 & 4,6 & 7,4 & 1,9 & 28,2 & 30,9 & 100,0 & 32,2 \\
\hline ES-3 & Estrella & 6,5 & 4,4 & 7,4 & 1,4 & 32,3 & 35,0 & 100,0 & 26,6 \\
\hline BA-4 & Bananita & 6,1 & 4,7 & 7,6 & 2,2 & 38,4 & 39,9 & 100,0 & 39,0 \\
\hline MO-5 & Moín & 5,8 & 3,8 & 7,8 & 1,2 & 29,3 & 30,8 & 100,0 & 40,7 \\
\hline MA-6 & Matina & 6,3 & 4,9 & 7,7 & 1,9 & 30,9 & 35,6 & 100,0 & 38,5 \\
\hline MA-7 & Matina & 6,0 & 3,8 & 7,5 & 1,7 & 31,5 & 29,7 & 96,3 & 33,5 \\
\hline MD-8 & Madre de Dios & 6,1 & 3,8 & 7,5 & 2,0 & 46,5 & 46,0 & 98,7 & 38,5 \\
\hline MD-9 & Madre de Dios & 5,6 & 3,8 & 7,4 & 1,4 & 43,6 & 38,8 & 90,8 & 43,4 \\
\hline PA-10 & Pacuare & 5,6 & 4,0 & 7,5 & 1,4 & 43,6 & 38,8 & 90,8 & 43,4 \\
\hline PA-11 & Pacuare & 7,9 & 6,7 & 8,1 & 1,1 & 25,8 & 39,4 & 100,0 & 35,5 \\
\hline PA-12 & Pacuare & 6,7 & 5,3 & 7,5 & 1,4 & 19,6 & 21,5 & 100,0 & 26,0 \\
\hline RE-13 & Reventazón & 6,4 & 4,8 & 7,4 & 1,4 & 19,3 & 20,1 & 100,0 & 28,3 \\
\hline RE-14 & Reventazón & 6,7 & 5,6 & 7,6 & 1,9 & 27,3 & 29,8 & 100,0 & 39,9 \\
\hline \multicolumn{10}{|c|}{ Caribe noroeste } \\
\hline RE-15 & Reventazón & 6,4 & 4,6 & 7,8 & 1,2 & 19,5 & 18,0 & 94,8 & 29,2 \\
\hline TO-16 & Tortuguero & 5,7 & 4,8 & 9,2 & 1,9 & 28,2 & 16,7 & 59,2 & 65,8 \\
\hline TO-17 & Tortuguero & 6,2 & 4,7 & 7,9 & 1,1 & 13,9 & 11,3 & 82,8 & 34,0 \\
\hline TO-18 & Tortuguero & 6,3 & 4,7 & 7,7 & 1,3 & 19,0 & 18,1 & 97,7 & 41,0 \\
\hline TO-19 & Tortuguero & 6,0 & 4,2 & 8,8 & 2,0 & 21,8 & 10,8 & 48,4 & 72,5 \\
\hline $\mathrm{CH}-20$ & Chirripó & 6,5 & 4,7 & 8,1 & 1,8 & 19,8 & 17,5 & 88,5 & 48,4 \\
\hline $\mathrm{CH}-21$ & Chirripó & 5,6 & 4,1 & 8,0 & 2,2 & 23,0 & 15,3 & 70,9 & 55,6 \\
\hline $\mathrm{CH}-22$ & Chirripó & 5,8 & 4,4 & 7,6 & 2,2 & 15,9 & 10,3 & 69,4 & 42,5 \\
\hline SA-23 & Sarapiquí & 6,5 & 4,6 & 8,2 & 2,3 & 26,3 & 19,3 & 73,6 & 63,9 \\
\hline SA-24 & Sarapiquí & 5,8 & 4,4 & 9,6 & 3,0 & 22,4 & 12,7 & 56,7 & 71,2 \\
\hline \multicolumn{2}{|c|}{ Media general } & 6,3 & 4,7 & 7,8 & 1,7 & 26,6 & 25,9 & 88,0 & 41,7 \\
\hline \multicolumn{2}{|c|}{ Media del Sureste } & 6,3 & 4,6 & 7,6 & 1,7 & 32,0 & 33,7 & 98,3 & 36,2 \\
\hline \multicolumn{2}{|c|}{ Media del Noroeste } & 6,1 & 4,5 & 8,3 & 1,9 & 21,0 & 15,0 & 74,2 & 52,4 \\
\hline \multicolumn{2}{|c|}{ Valor Mínimo ${ }^{2}$} & 4,1 & 3,1 & 7,2 & 0,2 & 7,5 & 3,8 & 20,8 & 6,6 \\
\hline \multicolumn{2}{|c|}{ Valor Máximo $^{2}$} & 7,9 & 6,9 & 10,6 & 8,2 & 54,0 & 54,9 & 100,0 & 99,1 \\
\hline
\end{tabular}

${ }^{1 /}$ Se refiere al promedio de cada característica en todos aquellos horizontes estudiados. Fuente: Cuadro 3.

${ }^{2 /}$ Referido mínimos y máximos del Cuadro 3.

de 7,5 cmol. $\mathrm{l}^{-1}$ y un máximo de 54 cmol. $\mathrm{l}^{-1}$. Los valores menores de CIC se encontraron en suelos del noroeste, con una media de $21 \mathrm{cmol}^{-1} \mathrm{l}^{-1}$, y los valores mayores en los suelos sureste, en donde la media fue de $32 \mathrm{cmol.1}^{-1}$, lo cual se considera normal debido al diferente origen geológico de los suelos de cada zona.

Los suelos con las menores CIC fueron el Fluventic Endoaquepts, perfil 4, el Fluvaquentic Eutrudepts, perfil 5 y el Andic Eutrudepts perfil 3 , todos de noreste; y los suelos con las mayores CIC fueron los Fluvaquentic Eutrudepts, perfiles 2 y 3 y el Fluventic Endoaquepts, perfil 3 todos de la zona sureste. En parte, los valores bajos de CIC en los suelos del noroeste pueden deberse a su textura más gruesa; lo opuesto ocurre en la zona sureste por la presencia de arcillas 2:1 (López 1963, Hardy 1970, Stotzky y Torrence 1963, Nieuwenhuyse 1996).

La suma de bases $(\Sigma \mathrm{B})$, o la capacidad de intercambio de cationes efectiva (CICE), siguen un comportamiento similar a la CIC. Su valor promedio para la región es de $25,9 \mathrm{cmol}^{-1} \mathrm{l}^{-1}$, con un mínimo de $3,8 \mathrm{cmol.}^{-1}$ y un máximo de 54,9 cmol. $1^{-1}$. Los suelos del noroeste presentaron en promedio una $\Sigma \mathrm{B}$ de $15 \mathrm{cmol}^{-1} \mathrm{l}^{-1}$ y los suelos del sureste de 33,7 cmol.1 ${ }^{-1}$. En ambas secciones, aunque con diferente intensidad la $\Sigma \mathrm{B}$ estuvo ligada a la textura y origen del suelo, de tal forma que los suelos más arcillosos presentaros las mayores $\Sigma \mathrm{B}$. 
Los suelos con las mayores $\Sigma \mathrm{B}$ fueron el Fluvaquentic Epiaquepts, el Fluvaquentic Eutrudepts, perfiles 1-2, el Fluventic Endoaquepts, perfil 3 y el Typic Udifluvents todos del sureste y los de menor $\Sigma \mathrm{B}$ fueron el Fluvaquentic Eutrudepts, perfil 5, el Fluventic Dystrudepts y los Andic Eutrudepts, perfiles 3 y 5 .

La saturación de bases presentó un comportamiento similar a la $\Sigma \mathrm{B}$ en ambas secciones; el valor medio para todos los suelos fue de $88,0 \%$, con un mínimo de $20,8 \%$ y un máximo de $100 \%$. Los elevados valores de saturación de bases, no reflejan las condiciones de meteorización de la región, a no ser que se considere el drenaje reducido de grandes extensiones de la misma, la disolución de bases a partir de materiales primarios y la adición de bases como enmiendas. El valor máximo correspondió al Typic Udifluvents en donde se detectaron carbonatos de calcio, probablemente fracciones de coral (oolitos) de antiguos frentes de playa redistribuidos de forma aluvial. De todos los suelos, solamente el Typic Hapludands y el Fluventic Dystrudepts presentaron saturaciones de bases menores al $60 \%$ entre 25 y $75 \mathrm{~cm}$ de profundidad lo que permitió clasificarlos como dístricos; por el contrario el resto de los suelos presentó, en al menos un horizonte de 25 a $75 \mathrm{~cm}$, más de $60 \%$ de saturación de bases por lo que se clasificaron como éutricos. De acuerdo con los rangos de interpretación de Bertsch (1986) todos los suelos presentan sumas de bases medias y altas saturaciones de bases en los primeros $60 \mathrm{~cm}$ de profundidad lo cual es sinónimo de alta fertilidad química.

La materia orgánica $(\mathrm{MO})$ presentó un valor medio de $1,7 \%$, con un mínimo de 0,2 y un máximo de 8,2. En general, en la mayoría de suelos la mayor cantidad de MO se presentó en el horizonte superficial. La irregularidad del contenido de MO con la profundidad de 18 suelos evidencia su origen a partir de deposiciones aluviales frecuentes; otros 6 suelos presentaron una disminución regular del contenido de MO, lo que evidencia que las deposiciones no son tan frecuentes. En general, los suelos con propiedades ándicas del noroeste presentaron cantidades de MO más elevadas en los primeros $40 \mathrm{~cm}$ que los Inceptisoles del sureste, hecho asociado a la formación de complejos organo-minerales en Andisoles de Costa Rica (Alvarado et al. 2001) y en la región Atlántica (Nieuwenhuyse y Kroonenberg 1994). El contenido de MO de toda la región es relativamente bajo lo que se asocia a la elevada temperatura del suelo (Sauerbeck y González 1977) y a la facilidad de descomposición de los residuos de banano (Vargas y Flores 1995).

\section{Clasificación taxonómica}

Los suelos bananeros de las 2 zonas se clasificaron en 3 ordenes, 22 como Inceptisoles, un Andisol y un Entisol (Cuadro 5). Para toda la llanura aluvial del Caribe, esta situación ha sido observada en estudios anteriores (Jiménez 1972, Luijckx y Zunnenberg 1992, Nieuwenhuyse 1996); sin embargo, la abundancia de Entisoles en áreas de inundación actual o la de Andisoles en fincas con mucha influencia volcánica suele ser mayor (Sancho et al. 1989, Stoorvogel y Eppink 1995).

Los Inceptisoles incluyen los subgrupos Epiaquepts, Endoaquepts, Eutrudepts y Dystrudepts. Los Aquepts, presentaron condiciones de mal drenaje a menos de $50 \mathrm{~cm}$ de profundidad, en forma continua o estratificada desde el límite de saturación; lo que permitió separarlos en los grandes grupos Endoaquepts y Epiaquepts. A nivel de subgrupo con excepción del Aquandic Endoaquepts el resto de los Aquepts presentó decrecimientos irregulares y contenidos de $\mathrm{MO}$ mayores a 0,2\% lo que los agrupó como Fluventic.

Los Eutrudepts presentaron régimen de humedad perúdico y saturación de bases mayor a $60 \%$ entre 25 y $75 \mathrm{~cm}$ de profundidad; lo cual se debe a que se originaron a partir de materiales sedimentarios altos en bases y a la ausencia de períodos secos mayores a 90 días acumulativos. Los Dystrudepts presentaron régimen perúdico y saturación de bases menor a $60 \%$ en al menos un horizonte, lo cual está asociado a la menor fertilidad de los materiales volcánicos que dieron su origen y al régimen de lluvias intensas de la región. Los Eutrudepts se agruparon como Andic, 


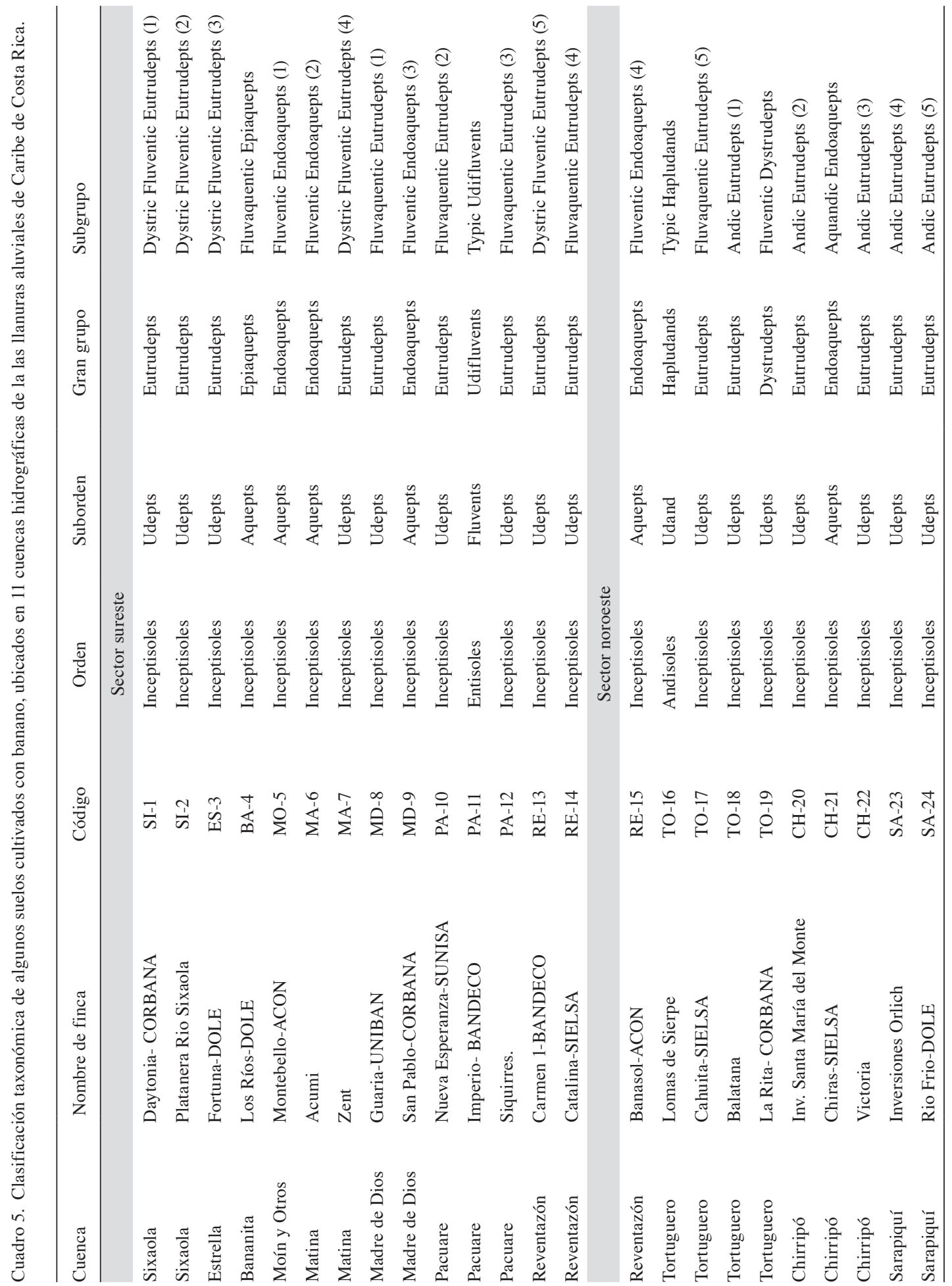


Fluvaquentic y Dystric Fluventic. Los subgrupos ándicos presentaron dentro de los primeros 75 $\mathrm{cm}$ de suelo, uno o más horizontes con un grosor total mayor a $18 \mathrm{~cm}$ con una Da igual o menor a $1,0 \mathrm{Mg} \cdot \mathrm{kg}^{-1}$ y una suma total del $\%$ de Al más $1 / 2 \mathrm{Fe}$ (por oxalato de amonio) igual o mayor a 1. Los subgrupos Fluvaquentic presentaron decrecimientos irregulares y contenidos de MO mayores a $0,2 \%$ y condiciones áquicas asociadas a características redoximórficas y cromas de 2 o menos; mientras que los Dystric Fluventic no presentaron carbonatos libres ni condiciones áquicas. El subgrupo Dystrudepts al igual que otros Udepts presentó decrecimientos irregulares de MO, lo que permitió clasificarlo como Fluventic Dystrudepts.

El Andisol clasificó en el subgrupo Typic Hapludands, y se ubica en la finca Lomas de Sierpe. Se caracteriza por ser un suelo profundo, con drenaje bueno (horizontes Bg después de $90 \mathrm{~cm}$ ) de texturas medias y gruesas, de fertilidad media con un epipedón úmbrico, con características ándicas, sin vidrio volcánico y con un régimen de humedad údico.

El suelo clasificado como Typic Udifluvents se ubica en las márgenes del río Pacuare y se caracterizó por tener buen drenaje, texturas franca y franco arcillosa, alta fertilidad, decrecimiento irregulares de $\mathrm{MO}$ y régimen de humedad perúdico.

Básicamente se definen 2 grupos de suelos con propiedades químicas, morfológicas y taxonómicas diferentes. En el primer grupo de suelos ubicados en el sector Caribe noroeste y que abarcan las cuencas de los ríos Tortuguero, Chirripó, Sarapiquí y parte del Reventazón, se encontraron Inceptisoles con propiedades ándicas (Andic Eutrudepts, Aquandic Endoaquepts), Inceptisoles dístricos (Fluventic Dystrudepts) y Andisoles (Typic Hapludands). Estos suelos se caracterizaron por tener fertilidad media, drenaje bueno en sectores no depresionales, de media a alta fijación de fósforo, capacidad de intercambio de cationes media, saturación de bases media y posiblemente minerales de origen volcánico. El segundo grupo correspondió a Inceptisoles de origen aluvial (Fluventic Endoaquepts, Dystric Fluventic Eutrudepts, Fluvaquentic Epiaquepts, Fluvaquentic Eutrudepts), en su mayoría con altas concentraciones de bases (éutricos), nulas propiedades ándicas, y con problemas de drenajes en algunos casos (Endoaquepts, Epiaquepts). En la Figura 3 se muestran fotografías de perfiles típicos. Lara (1970), Jiménez (1972), López y Solís (1991), Luijckx y Zunnenberg (1992) y Nieuwenhuyse (1996) reportaron características similares para estos grupos de suelos. Geográficamente, Lara (1970), Jiménez (1972) dividieron estos grupos de suelos al este y oeste del río Reventazón; sin embargo al ser la cuenca del Reventazón tan amplia y de acuerdo con los resultados de esta investigación y a la geología propia de las partes montañosas, se considera que una mejor delimitación es dada a partir de la subcuenca conformada por los ríos Vueltas-Silencio-Parismina; de tal forma que dentro de la cuenca del rio Reventazón, hacia el noroeste de estos, se encuentran los suelos con influencia volcánica (Dechesne 1994) y hacia el sureste los suelos que no tienen influencia volcánica (Luijckx y Zunnenberg 1992). El río Vueltas nace en el costado noreste del volcán Turrialba, en el poblado de Acuífero, Guácimo, que está a 1500 msnm.

\section{CONCLUSIONES}

Los suelos estudiados se pueden agrupar por sus diferencias químicas, físicas y taxonómicas en 2 grupos, cuyo límite geográfico podría estár definido hacia el noroeste y sureste de los ríos Silencio, Vueltas y Parismina; los cuales pertenecen a la gran cuenca del río Reventazón.

Los suelos de las llanuras del noroeste se caracterizan en general por presentar buen drenaje, texturas medias (franco arenosa, franca, franca limosa), una suma de bases entre $10 \mathrm{y}$ $19 \mathrm{cmol}^{-1} \mathrm{l}^{-1}$, una CIC entre 13 y $28 \mathrm{cmol}^{-\mathrm{l}^{-1}}$, con propiedades ándicas en algunos suelos, carga permanente en arcillas y pedregosidad ocasional.

Los suelos de las llanuras de sureste se caracterizan en general por presentar problemas de drenaje en sectores más bajos, texturas finas 
y moderadamente finas (franco arcillosa, franco arcillosa limosa, arcillosa, arcillosa limosa), suma de bases entre 10 y $46 \mathrm{cmol}^{-1}{ }^{-1}$, CIC entre 19 y $46 \mathrm{cmol}^{-1} \mathrm{l}^{-1}$, sin propiedades ándicas, con carga permanente principalmente.

En suelos de la cuenca del Pacuare se encontraron carbonatos de $\mathrm{Ca}$ libres, lo cual explica los altos contenidos de este nutrimento en los suelos generados a partir de inundaciones de este río.

Desde el punto de vista taxonómico en las llanuras del noroeste los suelos predominantes son Inceptisoles y Andisoles de los grandes grupos Andic Eutrudepts, Aquandic Endoaquepts, Fluventic Dystrudepts, Typic Hapludands y Fluventic, Dystric Fluventic, Fluvaquentic Eutrudepts.

Desde el punto de vista taxonómico en las llanuras del sureste los suelos predominantes son Inceptisoles de los grandes grupos Endoaquepts, Epiaquepts y Fluvaquentic Eutrudepts.

\section{LITERATURA CITADA}

ALVARADO A. 2006. Potential of soil carbon secuestration in Costa Rica, pp. 147-161. In: R. Lal, C.C. Cerri, M. Bernoux, J. Etchevers, C.E. Cerri (eds). Carbon secuestration in soils of Latin America. Chapter 8. The Haworth Press Inc. USA.

ALVARADO A., BERTSCH F., BORNEMISZA E., CABALCETA G., FORSYTHE W., HENRÍQUEZ C., MATA R., MOLINA E., SALAS R. 2001. Suelos derivados de cenizas volcá nicas (Andisoles) de Costa Rica. Asociación Costarricense de la Ciencia del Suelo y Centro de Investigaciones Agronómicas/ Universidad de Costa Rica. San José, Costa Rica. $111 \mathrm{p}$.

ALVARADO A., FORSYTHE W. 2005. Variación de la densidad aparente en órdenes de suelos de Costa Rica. Agronomía Costarricense 29(1):85-94.

ARAYA M., VARGAS A., CHÉVEZ A. 1998. Changes in distribution of roots of banana (Musa AAA, $\mathrm{Cv}$ Valery) with plant height, distance from the pseudostem, and soil depht. Journal of Horticulture Science \& Biotecnology 73(4):437-440.

ARAYA M., BLANCO F. 2001. Changes in the stratification and spatial distribution of the banana (Musa AAA, $\mathrm{Cv}$ Gran Naine) root system of poor, regular and good developed plants. Journal of Plant Nutrition 21(11):1679-1693.

BERTSCH F. 1986. Manual para interpretar la fertilidad de los suelos de Costa Rica. San José, Costa Rica. Oficina de Publicaciones. UCR. 76 p.

BENNETT H.H. 1926. General soil regions of eastern and northern Costa Rica, pp. 66-83. In: Treadwell, Jh. Cl. Possibilities for rubber production in Northern Tropical America. Washington, US Bureau of Foreign and Domestic Commerce, 1026.

CHAPMAN H.D. 1965. Cation-exchange capacity, pp. 814 901. In: C.A. Black ed. Methods of soil analysis: Chemical and microbiological properties. Madison, Wiscousin, American Society of Agronomy. Agronomy Series No. 9, part 2.

COHEN A.D., RAYMOND R., MORA S., ALVARADO A., MALAVASSI L. 1986. Características geológicas de los depósitos de turba en Costa Rica (estudio preliminar). Rev. Geol. Amer. Central 4:47-67.

CORBANA (CORPORACIÓN BANANERA NACIONAL) 2009. Estadísticas Bananeras. San José Costa Rica. Consultado el 25 de septiembre 2009. Disponible en http//www.corbana.co.cr/est_industria.shtml.

DECHESNE M. 1994. Island-arc volcanism and episodic fluvial sedimentation in the Atlantic Zone of Costa Rica. CATIE/AUW/MAG. Atlantic Zone Programme. Report N ${ }^{\circ}$. 91. Field Report No. 137. Costa Rica. sp.

DÓNDOLI C., DENGO G., MALAVASSI E. 1968. Mapa geológico de Costa Rica. San José, Dirección de Geología, Minas y Petróleo. Esc. 1:700000 color.

GUGGENBERGER G., ZEC W. 1999. Soil organic matter composition under primary forest, pasture, and secondary succession, Region Huetar Norte, Costa Rica. Forest Ecology and Management 124(1):93104.

HARDY F. 1970. Suelos Tropicales. Pedología tropical con énfasis en suelos de América. Editorial Herrero Hermanos, México.1era ed. 270 p.

HOLDRIDGE L.R. 1996. Ecología basada en zonas de vida. 4ta reimpresión. San José, Costa Rica. IICA. 216 p.

JARAMILLO R., VÁSQUEZ A. 1980. En cultivo de banano ASBANA propone sistema para clasificar aptitud de tierras. ASBANA 4(12):11-15. 
JIMÉNEZ T. 1972. Génesis, clasificación y capacidad de uso de algunos suelos de la Región Atlántica de Costa Rica. Tesis de Ingeniero Agronómo, Universidad de Costa Rica, San José, Costa Rica. 180 p.

LARA F. 1970. Problemas y procedimientos bananeros en la zona Atlántica de Costa Rica. San José, Costa Rica. Hermanos Trejos. pp.11-12.

LÓPEZ C.A. 1963. Identificación y clasificación de los minerales de arcilla presentes en nueve suelos de Costa Rica. Tesis de Ingeniero Agronómo, Universidad de Costa Rica, San José, Costa Rica. $56 \mathrm{p}$.

LÓPEZ A., SOLÍS P. 1991. Contenido e interacciones de los nutrimentos en tres zonas bananeras de Costa Rica. CORBANA 15(36):25-32.

LUIJCKX F., ZUNNENBERG W. 1992. Geomorphology and soils of the areas Limón-Cahuita, Atlantic Zone of Costa Rica. Report. Centro Agronómico Tropical de Investigación y Enseñanza/Atlantic Zone Programme (Costa Rica). No. 5. 55 p.

MORA R. 2007. Suelos expansivos de la ciudad de Limón, Costa Rica: Caso urbanización Quiribrí. Proyecto Fundevi 0960. Servicios especializados de Laboratorio de Suelos y Rocas (SELSYR) FUNDEVI, Universidad de Costa Rica. 28 p.

NATIONAL SOIL SURVEY CENTER. 2002. Field book for describing and sampling soils (Version 2.0). Washington, USA. Natural Resources Department of Agriculture. United States, Department of Agriculture.

NELSON D.W., SOMMERS L.E. 1982. Total carbon, organic carbon, and organic matter, pp. 539-279. In: A.L Page, R.H. Miller RH, R. Keeney (eds). Methods of soil analysis: Chemical and microbiological properties. Madison, Wisconsin, American Society of Agronomy. 2 ed. Agronomy Series Nº. 9, Part 2.

NIEUWENHUYSE A. 1996. Soils, geology and soil related sustainability aspects of the perhumid tropical Limon basin, Costa Rica. Guápiles, Costa Rica, CATIE. Serie técnica No. 272, 80 p.

NIEUWENHUYSE A., JONGMANS A.G., van BREMEN N. 1994A. Mineralogy of a Holocene chronosequence on andesitic beach sediments in Costa Rica. Soil Sci. Soc. Am. J. 58:485-494.

NIEUWENHUYSE A., JONGMANS A.G., van BREMEN N. 1994B. Andisol formation in a Holocene beach ridge plain under humid climate of the Atlantic coast of Costa Rica. Geoderma 57:425-442.

NIEUWENHUYSE A., KROONENBERG S.B. 1994 Volcanic origin of Holocene beach ridges along the Caribbean coast of Costa Rica. Marine Geology 120:13-26.

NIEUWENHUYSE A., vam BREMEN N. 1997. Quantitative aspects of weathering and neoformation in selected Costa Rican volcanic soils. Soil Science Society of America Journal 61:1450-1458.

NUHN H., PEREZ S. 1967. Estudio geográfico regional. Zona Caribe Norte de Costa Rica. Instituto de Tierras y Colonización (ITCO). San José, Costa Rica. 360 p.

OLSEN S.R., SOMMERS L.E. 1982. Phosphorus, pp. 403-430. In: A.L. Page, R.H. Miller, R. Keeney (eds). Methods of soil analysis: Chemical and microbiological properties. Madison, Wiscousin, American Society of Agronomy. 2ed. Agronomy Series $N^{\circ} .9$, part 2.

POWERS J.S. 2001. Geographic variation in soil organic carbon dynamics following land-use change in Costa Rica. Ph. D. Duke University, Graduate School and Management of Biology, Durham, N.C. 281 p.

POWERS J.S. 2004. Changes in soil carbon and nitrogen after contrasting land-use transitions in northeastern Costa Rica. Ecosystems 7(2):134-146.

POWERS J.S., SCHLENSINGER W.H. 2002A. Geographic and vertical patterns of stable carbon isotopes in tropical rain forest soils of Costa Rica. Geoderma 109(1-2):140-160.

POWERS J.S., SCHLENSINGER W.H. 2002B. Relationships among soil carbon distributions and biophysical factors at nested spatial scales in rain forest of northern Costa Rica. Geoderma 109(3-4):165-190.

PRESCOTT S.C. 1918. Examination of tropical soils. United Fruit Company. The Research Laboratory. Bulletin No. 3; 594 p.

RUSSELL A.E., CAMBARDELlA C.A., EWELL J.J., PARKIN T.B. 2004. Species, rotation, and lifeform diversity effects on soil carbon in experimental tropical ecosystems. Ecological Applications 14(1):47-60.

SANCHO F., MATA R., MOLINA E., SALAS R. 1989. Estudio de suelos de la finca de la Escuela de 
Agricultura de la región Tropical Húmeda, Guácimo, Limón. San José Costa Rica. 151 p.

SAUERBECK D.R., GONZÁLEZ M.A. 1977. Field decomposition of carbon-14-labelled plant residues in various soils of the Federal Republic of Germany and Costa Rica, pp. 159-170. In: IAEA and FAO (eds.) in cooperation with Agrochimica Vol. 1. "Proc. Symp. Braunschweig".

SERRANO E. 2002. Diagnóstico físico-químico del suelo y su relación con el crecimiento del banano en fincas con diferentes condiciones edafoclimáticas de la zona Caribe de Costa Rica, pp. 113-117. In: Informe anual 2002, Dirección de Investigaciones CORBANA (CORPORACION BANANERA NACIONAL). San José, Costa Rica.

SERRANO E. 2005. Banana soil acidification in the Caribbean coast of Costa Rica and its relationship with increased aluminium concentrations, pp. 142148. In: D. Turner and F. Rosales (eds). Banana Root System: towards a better understanding for its productive management. Proceedings of an international symposium held in San José, Costa Rica, 3-5 November 2003.
STOTZKY G., TORRENCE R. 1963. Soil mineralogy in relation to spread of fusarium wilt of banana in Central America. Plant and Soil 16(3):317-337.

STOORVOGEL J., EPPINK G. 1995. Atlas de la zona Atlántica Norte de Costa Rica. Guápiles, Costa Rica. Programa Zona Atlántica (CATIE/UAV/MAG).

SOIL SURVEY STAFF. 2006. Keys to Soil Taxonomy. 10th Edition, Washington, USA. Natural Resources Department of Agriculture. United States, Department of Agriculture. 321 p.

van DAM D., VELDKAMP E., van BREEMEN N. 1997. Soil organic carbon dynamics: variability with depth in foreted and deforested soils under pasture in Costa Rica. Biogeochemistry 39(3):343-375.

VARGAS G. 2004. La escala zonal, regional y local en el análisis del régimen pluviométrico del istmo Centroamericano. Revista Reflexiones 83 (1):31-56.

VARGAS R., FLORES C.L. 1995. Retribución nutricional de los residuos de hojas, venas de hojas, pseudtallo y pinzote de banano (Mussa AAA) en fincas de diferentes edades de cultivo. Revista CORBANA 20(44):33-47. 